\title{
Phase retrieval from carrier frequency interferograms: reduction of the impact of space-variant disturbances
}

J. Schwider

Johannes.Schwider@physik.uni-erlangen.de

\section{Nercissian}

K. Mantel

\author{
Institute of Optics, Information and Photonics, University Erlangen-Nuremberg, Staudtstr. 7/B2, 91058 \\ Erlangen, Germany \\ Institute of Optics, Information and Photonics, University Erlangen-Nuremberg, Staudtstr. 7/B2, 91058 \\ Erlangen, Germany \\ Max Planck Institute for the Science of Light, Staudtstr. 7/B2, 91058 Erlangen, Germany
}

Phase "extraction" by using temporal phase shifting is sensitive to vibrations and drifts, producing systematic phase errors periodic with twice the fringe frequency. This error source may be avoided by evaluating only single carrier frequency interferograms, which makes the procedure immune against vibrations and drifts provided that the integration time is short enough to freeze the fringe pattern. However, the phases extracted from single interferograms in this way often show local irregularities depending on the mean phase of the interference pattern. Such local phase irregularities are caused by local disturbances in the light path like specks and dust particles on the optical components of the interferometer. Moreover, since digitized data are gathered, there is a nonlinear processing step involved which is also responsible for the generation of such irregularities. Here, it is proposed to use a set of suitably combined phase-ramped interferograms to reduce phase dependent irregularities. The proposed averaging technique also reduces edge ringing effects known from Fourier evaluation procedures. Since the imaging optics also contributes to the phase to be measured when tilted wavefronts are used, calibration is mandatory. The calibrated state is only valid if strict rules considering fringe number per diameter as well as the position of the wedge in the interferometer are maintained in the measuring process.

[DOI: http://dx.doi.org/10.2971/jeos.2015.15003]

Keywords: Interferometry, phase retrieval, carrier frequency, synchronous detection

\section{INTRODUCTION}

The viability of interferometric methods strongly depends on the immunity against environmental disturbances like vibrations or drifts of the phase to be measured. This requires short acquisition times for the interference patterns in order to freeze the phase. Here, the use of carrier frequency fringe patterns comes to the fore which means that the phase is contained in a single fringe pattern which has to be evaluated. Before the advent of array detectors and PCs fringe positions were determined using photometric methods [1]. Only few fringes per diameter were used to keep systematic errors within predetermined limits. A great variety of fringe evaluation methods [2]-[6] were developed over the years comprising fringe follower software of electronically detected interferograms, extraction of the phase via Fourier transform procedures and next neighbourhood operations as spatial phase shifting or synchronous detection. Especially the most modern methods are using high spatial frequency fringe patterns [7]-[10]. The main emphasis rests on evaluations with short exposures avoiding degradations of fringe contrast and averaging of a big number of measured phase distributions in order to reach sufficient repeatability for the test of high quality optics.

\section{THE PHASE RETRIEVAL PROCESS}

Here we want to discuss fringe evaluations for interference patterns disturbed by small imperfections. An example for a typical interferogram is shown in Figure 1.

Considering the choice of the carrier frequency it is known from holography that the chosen carrier frequency limits the
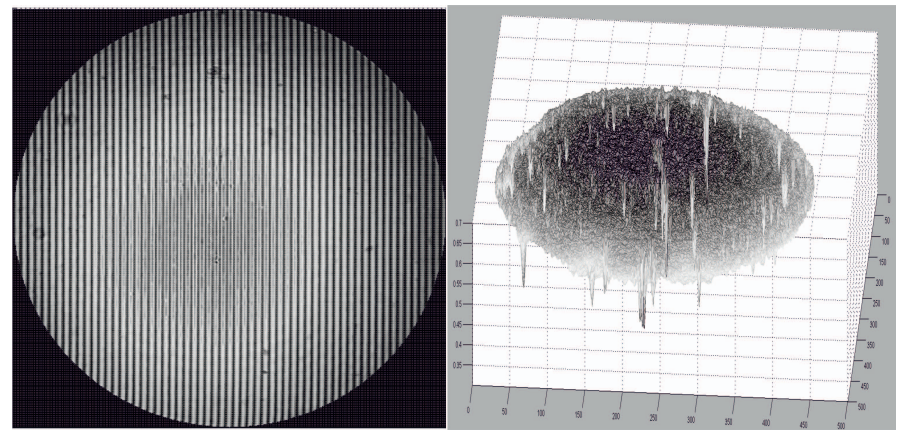

FIG. 1 Carrier frequency interferogram taken with a planar Fizeau interferometer having 60 fringes/diameter with bell-shaped illumination impaired by specks on the illuminating and imaging optics causing indentions of the fringe amplitude (on the left). On the right: local variations of the visibility of the fringe pattern due to specks and illumination variations (slanted view to show local blemishes more clearly). 
spatial resolution of the phase distribution to be measured. There is a bargain between the carrier frequency and other limitations set by the detector array and systematic errors caused by the imaging optics. The discrete detection of the intensity pattern limits the fringe period to more than 2 pixels and in practical terms to 4 pixels per period or more. In optical testing applications this gives a rather generous margin for the detectable spatial frequency content of the phase distribution resulting from surface or other deviations of a test piece in the interferometer. Furthermore, single frame evaluations allow for averaging of a big number of evaluated phase pictures minimizing all kinds of stochastic errors and even drifts caused through atmospheric variations of the optical path difference (OPD) in the interferometric cavity. Accessing a big number of interference patterns combined with a systematic ramp variation of the reference phase will give the best guarantee for an unambiguous phase average. State of the art CMOS or CCD-cameras allow for the acquisition of approx. 100 frames/sec with pixel-counts in the megapixel range provided the light source delivers sufficient power. In fact, such a procedure can be seen as a hybrid between temporal and spatial phase shifting interferometry which will be the main topic of this publication.

Let us assume that $M=2 N$ ( $N$ being a whole positive number) interference patterns have been recorded with integration times for each single frame in the order of $1 \mathrm{msec}$ and that during the grabbing of the interferogram set the reference phase $\varphi$ is tuned through $2 \pi$ (or multiples). The intensity distribution $I_{m}$ of a single interferogram shall be:

$$
\begin{aligned}
& I_{m}(x, y) \\
& =I_{0}(x, y)\left[1+V(x, y) \cos \left(\Phi(x, y)+2 \pi v_{c} x-\varphi_{m}\right)\right],
\end{aligned}
$$

where $\Phi(x, y)$ is the phase to be measured, $v_{c}$ is the carrier frequency adjusted in the interferometer and $\varphi_{m}=\frac{2 \pi m}{M}$ $(m=1 \ldots M)$ is the ramped reference phase. We choose here in a first approach synchronous detection for the phase extraction in order to demonstrate the advantage of difference interferograms in the phase retrieval process later on. Synchronous detection means that the data of the intensity pattern stored in the computer memory are multiplied with cosine and sinefunctions of a local oscillator provided via software having the carrier frequency $v_{s}$ :

$$
\begin{aligned}
& I_{m}(x, y) \cos \left(2 \pi v_{s} x\right) \\
= & I_{0}(x, y) \cos \left(2 \pi v_{s} x\right)+\left[I_{0}(x, y) V(x, y) \cos \left(2 \pi v_{s} x\right) \cos (\Phi(x, y)\right. \\
& \left.\left.+2 \pi v_{c} x-\varphi_{m}\right)\right] \\
= & I_{0}(x, y) \cos \left(2 \pi v_{s} x\right)+\frac{1}{2} I_{0}(x, y) V(x, y)[\cos (\Phi(x, y) \\
& \left.+2 \pi\left(v_{s}+v_{c}\right) x-\varphi_{m}\right)+\cos (\Phi(x, y) \\
& \left.\left.+2 \pi\left(v_{s}-v_{c}\right) x-\varphi_{m}\right)\right]
\end{aligned}
$$

and

$$
\begin{aligned}
& I_{m}(x, y) \sin \left(2 \pi v_{s} x\right) \\
= & I_{0}(x, y) \sin \left(2 \pi v_{s} x\right)+\left[I_{0}(x, y) V(x, y) \sin \left(2 \pi v_{s} x\right) \cos (\Phi(x, y)\right. \\
& \left.\left.+2 \pi v_{c} x-\varphi_{m}\right)\right] \\
= & I_{0}(x, y) \sin \left(2 \pi v_{s} x\right)+\frac{1}{2} I_{0}(x, y) V(x, y)[\sin (\Phi(x, y) \\
& \left.+2 \pi\left(v_{s}+v_{c}\right) x-\varphi_{m}\right)+\sin (\Phi(x, y) \\
& \left.\left.+2 \pi\left(v_{s}-v_{c}\right) x-\varphi_{m}\right)\right] .
\end{aligned}
$$

Under the assumption that the phase to be measured varies across the interferogram by about one fringe only and that the synthetic carrier frequency $v_{s}$ is close to $v_{c}$ then a low pass filter will let pass the low frequent contributions only which allow the calculation of the wrapped phase $\Phi$ :

$$
\begin{aligned}
& S \propto \frac{1}{2} I_{0}(x, y) V(x, y) \sin \left(\Phi(x, y)+2 \pi\left(v_{s}-v_{c}\right) x-\varphi_{m}\right) \\
& C \propto \frac{1}{2} I_{0}(x, y) V(x, y) \cos \left(\Phi(x, y)+2 \pi\left(v_{s}-v_{c}\right) x-\varphi_{m}\right) . \\
& \Phi(x, y)+2 \pi\left(v_{s}-v_{c}\right) x-\varphi_{m}=\arctan \frac{S}{C} .
\end{aligned}
$$

Since we have assumed that the interferograms of the set are phase shifted through $2 \pi$ the set can be split into two subsets being in phase opposition of the depth $2 \mathrm{~N}-1$.

The phase is extracted from the filtered side-band in the spatial frequency spectrum of the interferogram. The incoherent contribution to the interferogram intensity, or in electronical terminology the "dc-term", can be easily eliminated if two phase-shifted interferograms in phase opposition are subtracted from each other. This has two big advantages as will become clear below. The cancellation of the dc-offset will be maintained also in case of random phase shifts due to vibration or in case of drifts in the interferometer.

Now, the phase retrieval process can start with intensity difference values of the following kind:

$$
\begin{aligned}
\Delta I_{k}(x, y) & =I_{k+2^{N-1}}-I_{k} \\
& =2 I_{0}(x, y) V(x, y) \cos \left(\epsilon_{k}\right) \cos \left(\Phi+2 \pi v_{c} x\right),
\end{aligned}
$$

where $\epsilon_{k}$ symbolizes the mismatch of the reference phase difference from $\pi$ due to drifts and vibrations for the intensity difference $\Delta I_{k}$. As long as $\epsilon_{k}<\pi / 4$ the loss of modulation is tolerable. This condition should be met in most practical cases especially if the grabbing time for the whole set of interferograms is only a few seconds. The integration time of about $1 \mathrm{~ms}$ for a single interferogram should freeze the mechanical disturbances sufficiently. Since a discrete representation of the intensity distribution is processed it is advantageous to use 4 pixels/period or multiples of 4 . In this case, a period of the sine of the local oscillator can be represented by a vector [0 100 -10] for 4 pixels and by [0 $71070-7$-10 -7] for 8 pixels which alleviates the fast evaluation. In case of spatial phase shifting the corresponding convolution kernels are [0 10 - 0 -10] for the odd-kernel and [10 0 -10 0] for the even-kernel. In the latter approach the resulting phase pattern is periodic with the carrier frequency and has to be down-converted using the phase of the assumed ideal carrier. Similar operations can be 


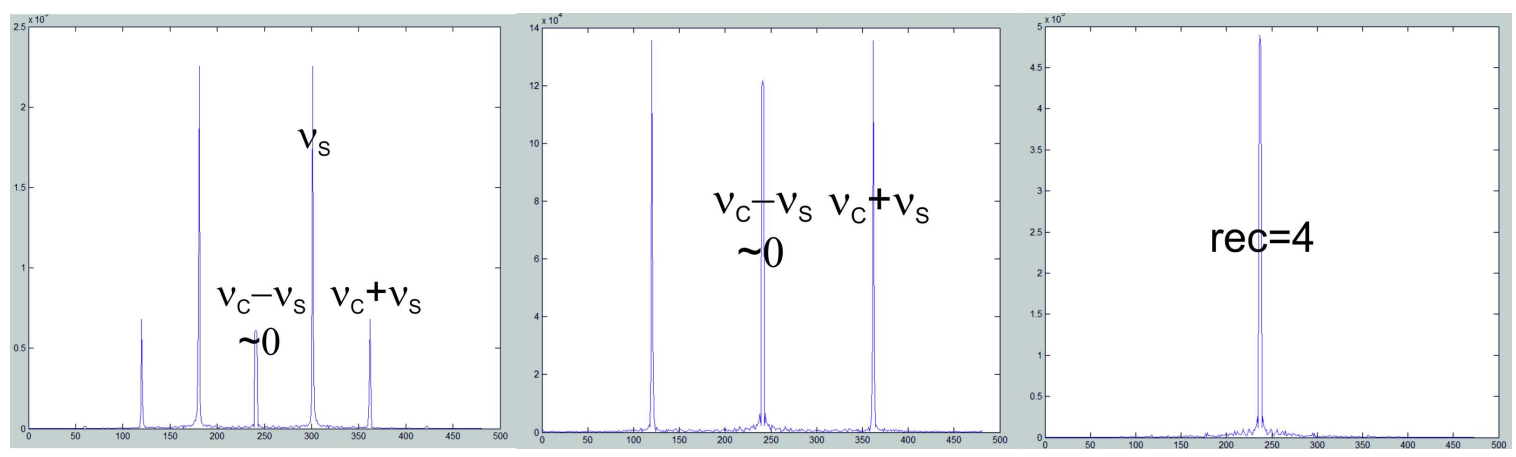

FIG. 2 Spatial frequency data of an interferogram with 60 fringes/diameter. On the left: FFT (Fast Fourier Transform) of the sine product for one of the fringe patterns, please note the presence of the dc-term multiplied with the sine of the local oscillator! Center: FFT of the sine product with the intensity difference of two interferograms being in phase opposition. Please note the absence of the dc-term! On the right: FFT of the difference interferograms convolved with a 4-pixel-wide rect-function resulting in the suppression of all disturbing harmonics.

carried out using the Fourier-method either delivering a highfrequency result or a low frequency phase distribution after a frequency shift of one of the side bands to zero.

The advantage of the use of such intensity differences becomes obvious in Fourier space. A comparison in the spatial frequency domain of the above intensity difference and the following synchronous detection steps with the intensity values having undergone the same retrieval process shows the improvement in the signal-to-interference ratio concerning all parasitic frequency components. For the difference intensities the carrier term is absent (see Figure 2) which reduces neighbourhood effects which are a basic feature of phase retrieval methods using single interferograms. In the case of synchronous detection only in the low pass filtering step the local neighbourhood is involved in the phase extraction process since the multiplication with the local oscillator does not couple the intensities of neighbouring pixels.

Time averages of many phase values require fast algorithms. Therefore, time-consuming unwrapping [10] algorithms should be avoided or be applied only after the averaging step. Unwrapping can be avoided if slope data are averaged. Then the time consuming integration of the slope data to the phase to be measured can be done a posteriori. Here we propose the phase addition in the mod-space by using the sine and cos-character of the quantities $S$ and C (Eq. (3)). Due to the synchronous detection procedure for each intensity difference $\Delta I_{k} S$ - and $C$-values have the same prefactors. If two such intensity differences have been processed in the mentioned manner two doublets $(S 1, C 1)$ and $(S 2, C 2)$ will result which can be added as given in Eq. (5):

$$
\Phi_{1}+\Phi_{2}=\arctan \left(\frac{S 1 C 2+S 2 C 1}{C 1 C 2-S 1 S 2}\right) .
$$

For a demonstration of the virtue of this averaging procedure we have used data sets which are produced by our phase shifting [11] Fizeau interferometer providing 6 intensity values with reference phase steps of $90^{\circ}$. For the evaluation, the software MATLAB has been applied delivering averaged phase values $\bmod (2 \pi)$. From the set of the intensity values (i1, i2,..., i6) four intensity differences i1-i3, i2-i4, i3-i5, i4-i6 can be calculated (see Figure 3 ). For the calculation of the av-

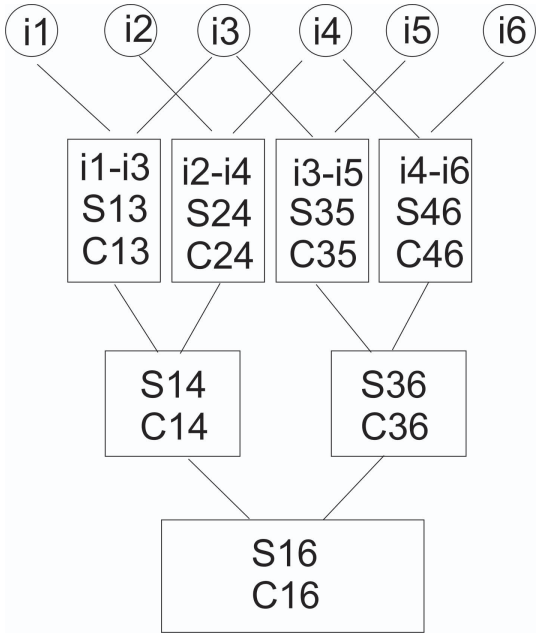

FIG. 3 Tree-diagram for the combination of 6 measured intensity values representing $\pi / 2$-phase shifted intensity values for the calculation of the sum phase $\bmod 2 \pi$.

eraged $\sin /$ cos-values $S 16$ may serve as an example:

$$
\begin{aligned}
S 16= & S 14 C 36+S 36 C 14 \\
= & (S 13 C 34+S 34 C 13)(C 35 C 46-S 35 S 46) \\
& +(S 35 C 46+S 46 C 35)(C 13 C 34-S 13 S 24) .
\end{aligned}
$$

The nominator S16 represents the sin-term showing a 4-fold phase sensitivity. The wrapped phase calculated in this manner for our demo is given in Figure 4 in comparison with a single phase result.

Repeatability measurements using two runs with 6 intensity values each show an improvement of the rms-figures for the unwrapped phase deviations by a factor of 1.7 which is a little smaller than the expected factor of 2 for the average of 4 phase measurements (see Figure 5).

The repeatability for the average has a rms-value of 0.0008 waves which is in the sub-Angstrom region. The wave aberration of the Fizeau combined with the systematic aberrations caused through the tilt and the achromatic doublet telescope including a folding mirror beside a pellicle beam splitter is several orders of magnitude greater.

Nevertheless, the correct tendency is indicated which fosters the realistic expectation that an increased number of averaged 


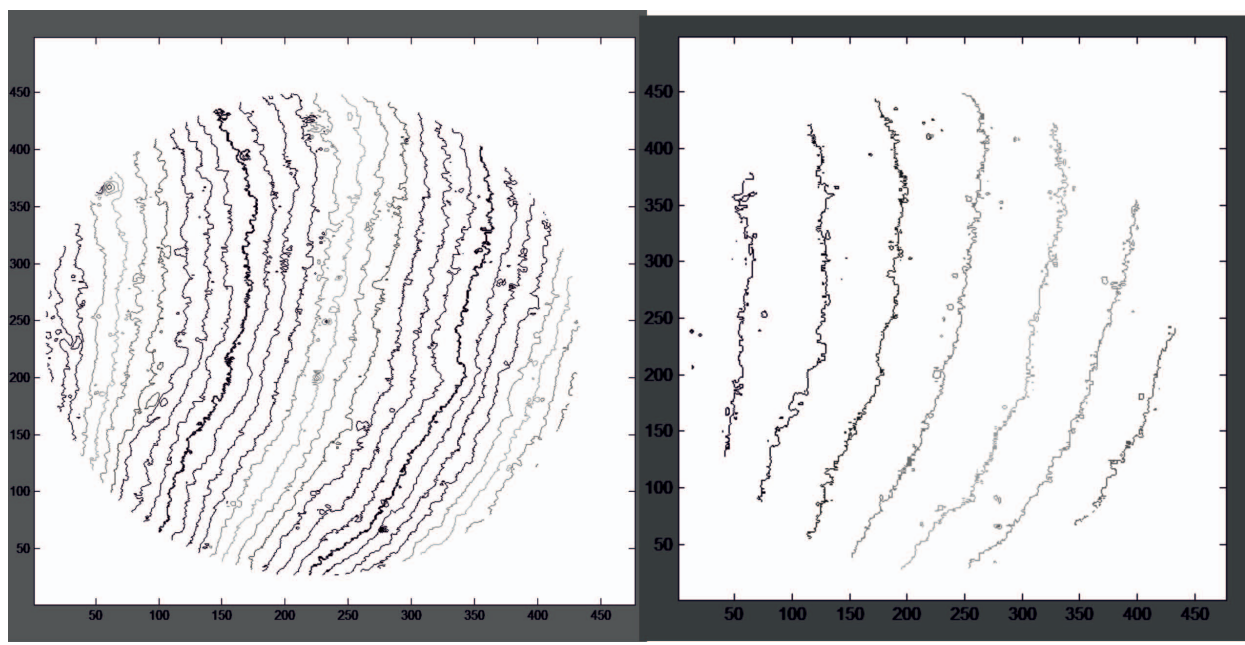

FIG. 4 Wrapped phase with 4 -fold sensitivity (on the left) against wrapped phase without summation due to Eq. (6).
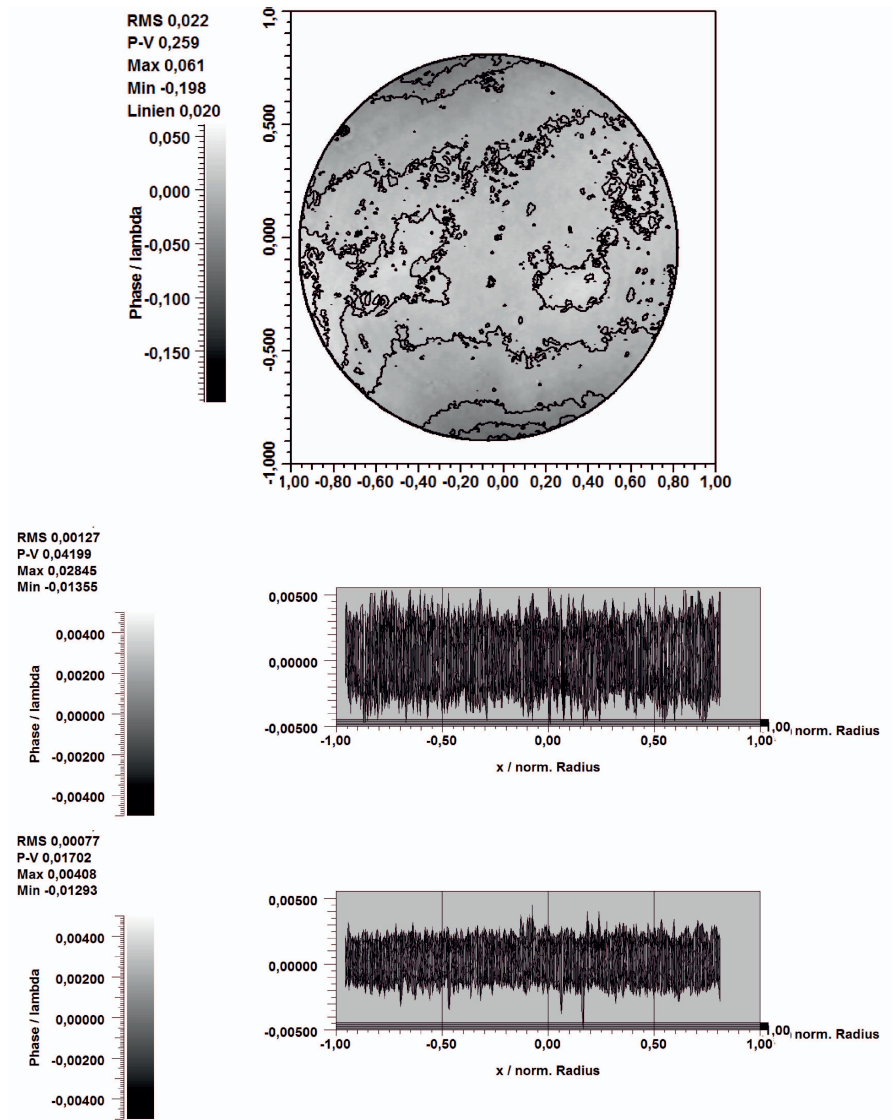

FIG. 5 Above: averaged phase data according to the diagram of Figure 3. Center: repeatability values for a single intensity difference, here, i1-i3. Below: repeatability values for the averaged data between two independent runs.

phase data will show even smaller rms-figures for the repeatability values. Generalizations of the averaging technique to a set of $2 M$ interferograms can make advantage of the power of two hierarchy in the averaging procedure as is indicated in Figure 6.

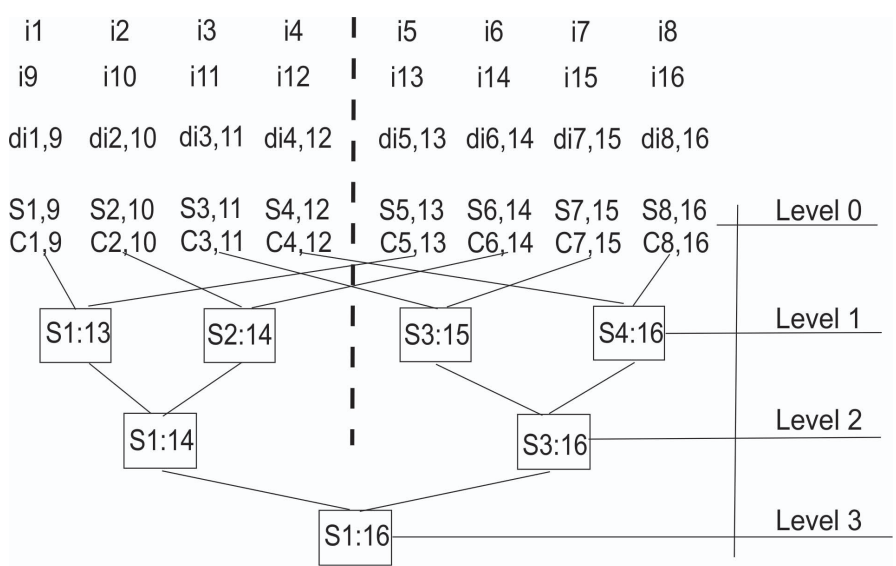

FIC. 6 Averaging tree for 16 intensity values, for the levels 1 to 3 only the sinusoidalvalues are depicted for simplicities sake. For 16 interferograms $M=16$ and $N=8$ which would result in a reduction of random errors by a factor $\sqrt{8}$. Further extensions in the depth of the data set and the averaging method are straightforward.

\section{IMPACT OF DUST DIFFRACTION PATTERNS ON THE REPEATABILITY}

Since between individual interferograms the mean phase will change, be it through piston movements or through slight tilts and also intentionally due to phase ramping, we have to look for the effect of such small variations on the repeatability of the evaluation process. For the demonstration of these influences we have used sets of 6 carrier interferograms obtained with the help of our phase shifting interferometer. Phase repeatability values can be obtained from calculating the phase difference of individual interferograms. There is a significant difference in the repeatability data for the phase difference of interferograms with the same mean phase status on the one hand and on the other with a phase change by fractions of one period. In Figure 7 such difference data are given for two interferograms being in phase and also in phase opposition.

It can be shown that within the set of the 6 phase-shifted interferograms the phase difference between interferograms i1 and $i 5$ behaves in a similar manner as the difference of two phases taken from two consecutive data-sets but with nearly identical mean phase status (see Figure 8). 


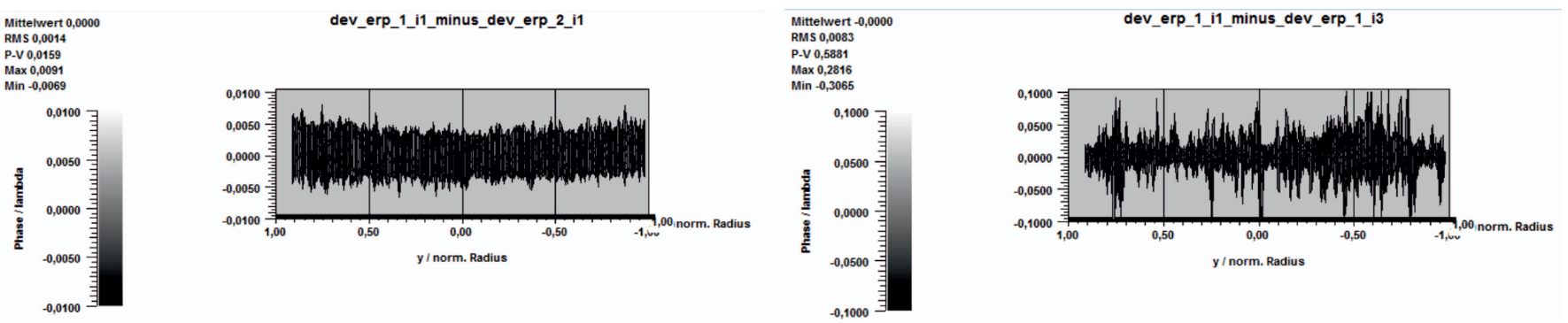

FIG. 7 On the left: phase difference of two interferograms having the same mean phase status. On the right: phase difference of two interferograms being out of phase by $180^{\circ}$.
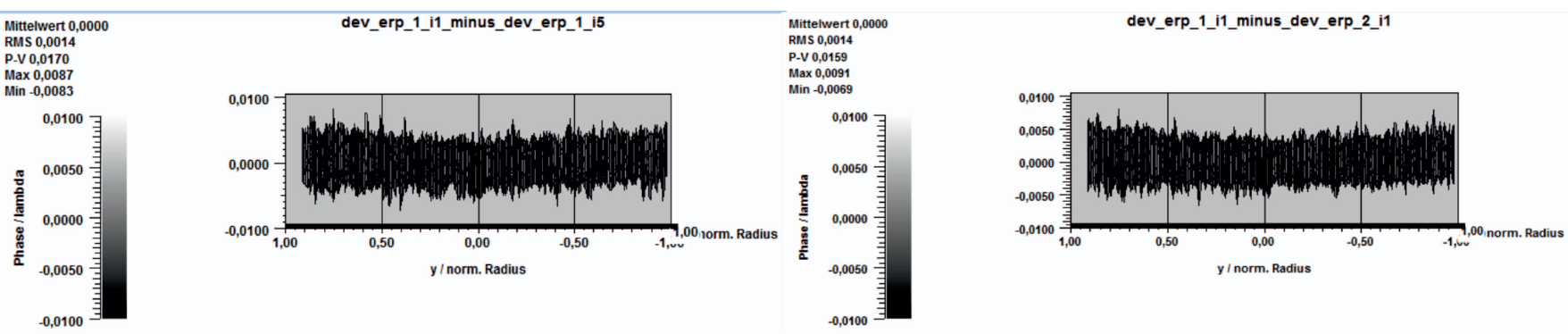

FIG. 8 On the left: phase difference of the interferograms i 1 and is of a single run. 0 n the right: phase difference of the interferograms i1_ 1 and i1_ 2 being the first interferograms each taken of two independent sets having the same mean phase.

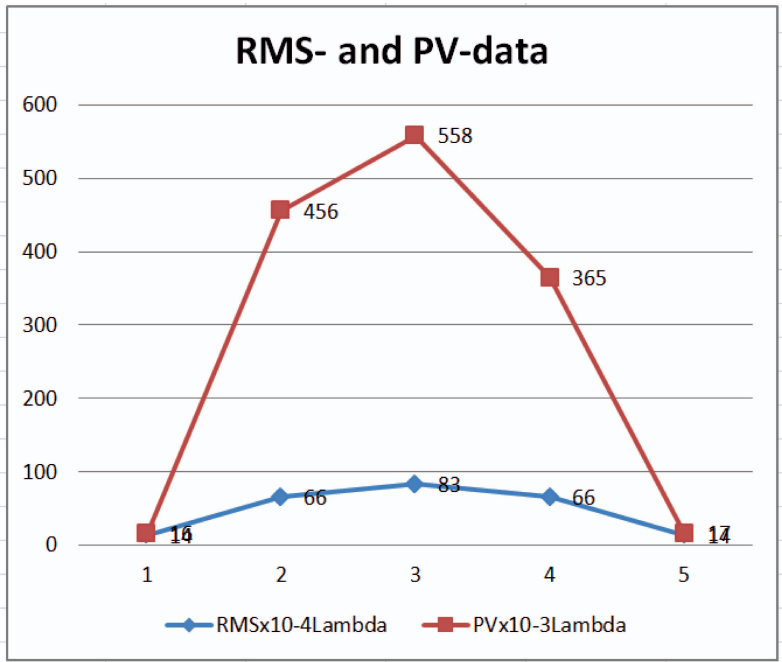

FIG. 9 rms- and pv-figures for the phase differences related to the phase of i1. On the abscissa: the numbers symbolize the differences with increasing mean phase offset, i.e. 2 corresponds with $90^{\circ}, 3$ corresponds with $180^{\circ}$ and so on in steps of $90^{\circ}$.

The dependence of the repeatability on the mean phase has been evaluated by subtracting the phase of interferogram i1 from all consecutive interferograms i2, i3, i4, i5 and for the interferogram i1 also beween the values resulting from two consecutive runs without changing the adjustment. The resulting rms- and pv-figures are summarized in Figure 9.

As mentioned before an improvement in the phase accuracy can be achieved if the incoherent bias or so-called dc-term is removed before processing the data. For this purpose one selects interference patterns being in phase opposition as e.g. i1 and $\mathrm{i} 3$ or i2 and i4 and calculates the intensity difference i1-i3 or i2-i4, respectively.

Furthermore, it is known from phase retrieval using the
Fourier-method after Takeda [2] that the phase in the rim region of the interferogram is disturbed through the spatial filter process. At first we want to discuss the impact of using difference interferograms. Due to the fact that the filtering process leads to a convolution of the phase data with a sinc- or Jinc-type function depending on the type of the filter function in Fourier-space, edge ringing effects will occur. Especially the convolution with the filter-function will contribute spurious phase contributions which will impair the wrapped phase date. Since we propose the phase retrieval from difference interferograms the dc-term is eliminated which reduces the edge ringing very much as can be inferred from Figure 10. In addition, the difference interferogram has an increased modulation depth which leads to a much smoother phase in the measuring field.

Now, we want to discuss the smoothing effect due to averaging of wrapped phase data retrieved from phase-ramped interferograms. The filtering process in case of the Fourier-phase retrieval is done by multiplying a rect-type filter function, here a circ-function. Therefore, in $(x, y)$-space a convolution with a Jinc-function is carried out. The convolution kernels have negative wings which produce together with the periodic interferogram function periodic disturbances in the rim region. However, the actual phase of the disturbances will be shifted together with the fringe pattern of the ramped interferograms. An averaged phase result will show a well defined phase rim comparable with temporal phase shifting results. In Figure 11, a single interferogram is contrasted to the wrapped data for an average of 4 interferograms.

\section{IMPACT OF THE IMAGING OPTICS ON THE MEASURED PHASE}

So far we have only dealt with the phase retrieval process which will not guarantee freedom from systematic aberrations 

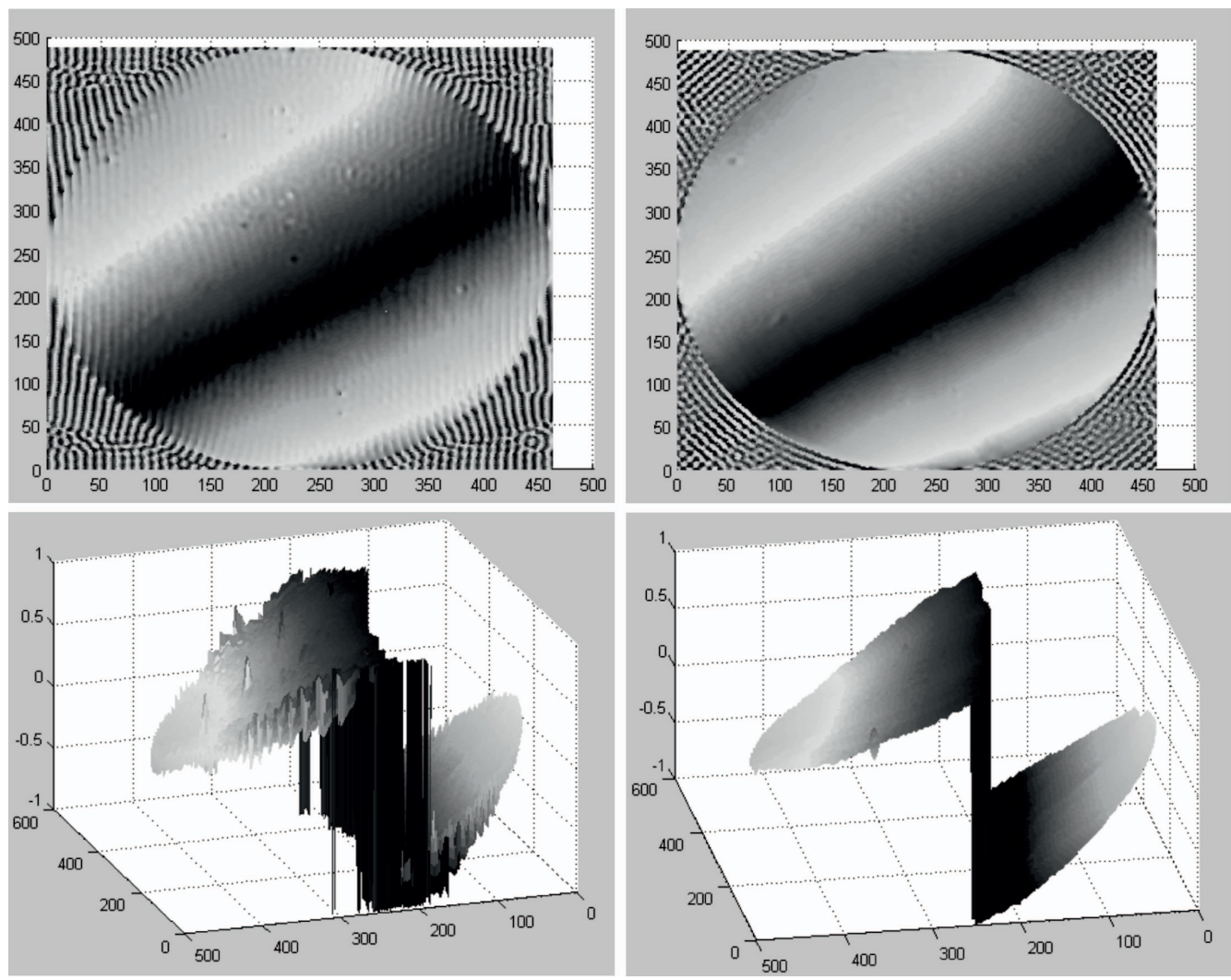

FIG. 10 Wrapped phase images resulting from a Fourier transform evaluation of interferograms with approx. 8 pixels/period using a circular frequency window with $r=55$. On the left: evaluation of a single intensity here iz. On the right: evaluation of a difference intensity iz-i5, please note the elimination of edge ringing and an improvement of the smoothness of the phase distribution.
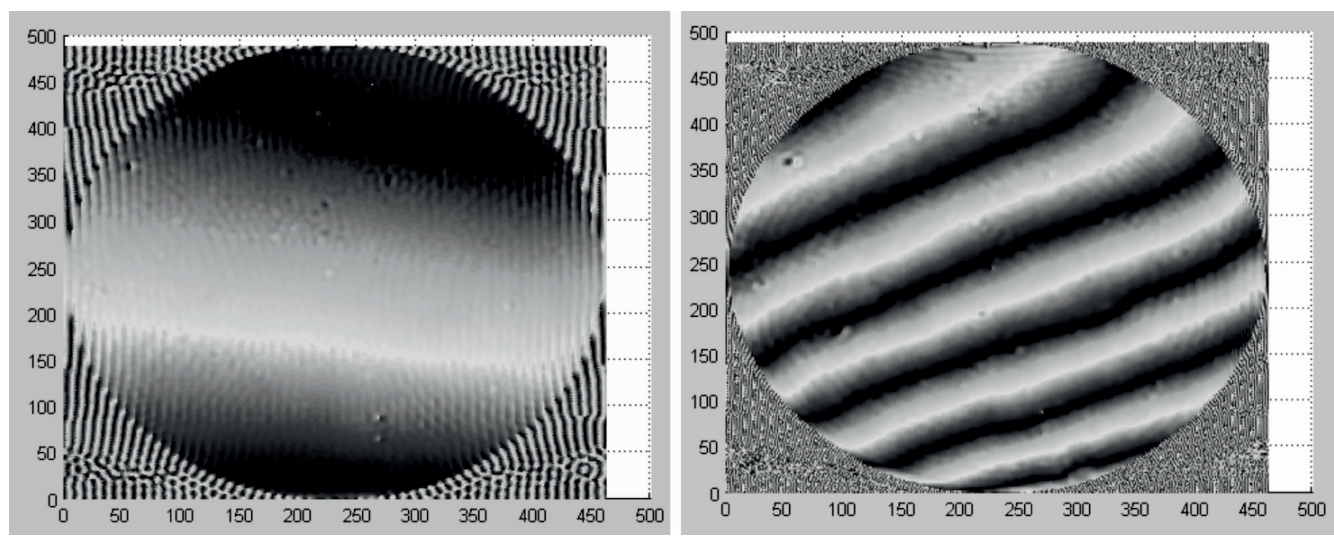

FIG. 11 Demonstration of the edge ringing reduction due to averaging. On the left: wrapped phase for single interferogram evaluation showing strong edge ringing. On the right: wrapped phase of the average of the phases of 4 interefrograms being ramped with $90^{\circ}$-phase steps.

caused by the optics of the interferometer. Such aberrations are the result of unequal light paths of the interfering waves through the imaging optics. Imaging systems based on achromatic doublets are a common solution compared with especially designed imaging systems. In any case, telescopic imaging systems are necessary to avoid hyperbolic fringes.

Ray-trace simulations [12] show that systematic wave aberrations of the order $\lambda / 20 \mathrm{rms}$ might occur. The strongest contributions result from defocus, primary astigmatism and coma. Therefore, two restrictions have to be obeyed: (1) calibration of the setup is mandatory and (2) in all following measurements the number of fringes should be kept within a corridor of 1 fringe/diameter in order to get reproducible results. However, in our experimental Fizeau setup the rotational sym- metry of the telescopic imaging system is broken since one plane folding mirror having deviations in the order of $\lambda / 10$ is used for practical reasons. Therefore, the symmetry of the aberrations for opposite wedge edge positions does not prevail which would be expected due to ray-trace [12] simulations.

Since the reference phase is ramped due to our proposal for phase retrieval it is self-evident to adjust fluffed out fringes in an additional run in order to determine the intrinsic wave aberrations for a chosen fringe frequency which can be used for correcting systematic errors (see Figure 12). If a calibrated reference surface is available the calibration of the interferometric setup is straightforward. The calibrated reference surface can of course be obtained if absolute measurements 

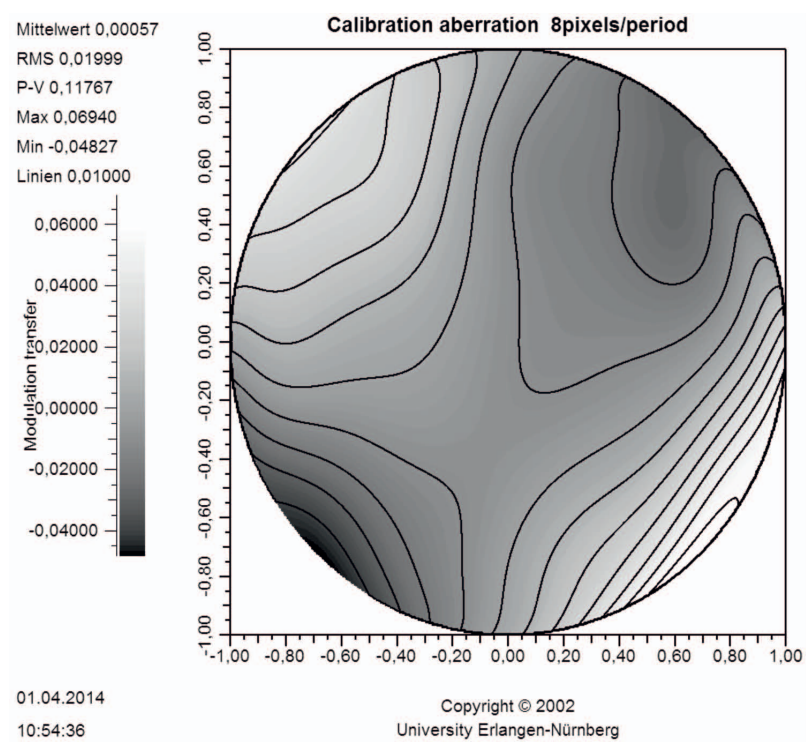

10:54:36
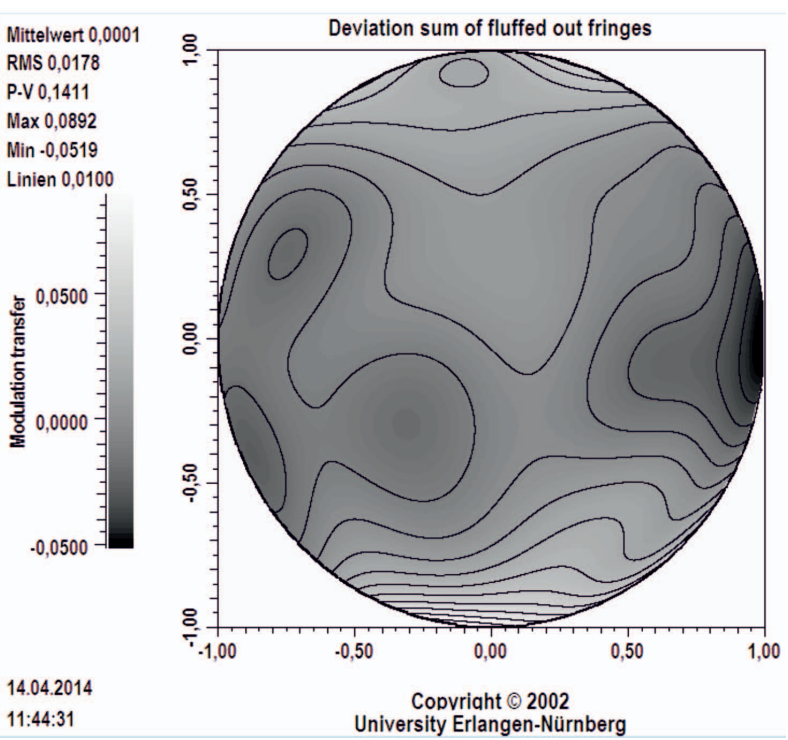

FIG. 12 Calibration function determined through calculating the difference between the deviations for 8 pixels/period and fluffed out fringes (left). On the right: deviations of the Fizeau resonator for fluffed out fringe field for a comparison.

would be made using the standard adjustment parameters for optimum repeatability.

\section{CONCLUSIONS}

We have investigated phase retrieval from single carrier frequency interferograms showing small space-variant defects due to imperfections of the optics or dust respective other local obstacles. These defects impair the retrieved local phase since all phase retrieval algorithms for single carrier frequency interferograms rely on next neighbour operations due to convolution with suitable even/odd kernels or low pass filtering. The accuracy of the retrieval process can be enhanced if a set of interferograms with ramped reference phases are combined. Since the phase modulates the coherent term of the intensity expression it is advantageous to subtract the intensities of two interferograms being in phase opposition. In this way one gets rid of the incoherent dc-term and obtains twice the modulation depth for the interference term. We have shown that following this concept the resulting phase distribution from any retrieval algorithms is considerably smoother even to the rim of the measuring aperture. Stochastic phase errors caused by electronic noise and other non-systematic errors are greatly reduced through averaging of many phase results. Averaging of phases resulting from phase-ramped interferograms improve also the definition of the phase distribution straight to the rim of the measured sample due to averaging phase-sensitive disturbances as edge ringing. Also, the systematic aberrations caused by the propagation of tilted waves through the imaging optics have been simulated for achromatic telescope optics. Since these aberrations are of the order of $1 / 20$ of a wavepv one has to calibrate the interferometer. After calibration the experimenter has to guarantee that the tilt is reproduced within one fringe per diameter for a tilt of 4 pixels/period. Therefore, adjustment helps are necessary to fulfill this condition for all following-on measurements.

\section{ACKNOWLEDGEMENTS}

The financial support by the Max Planck Institute for the Science of Light is gratefully acknowledged.

\section{References}

[1] G. D. Dew, "A method for the precise evaluation of interferograms," J. Sci. Instrum. 41, 160-162 (1964).

[2] H. I. Takeda, and S. Kobayashi, "Fourier-transform method of fringe-pattern analysis for computer-based topography and interferometry," J. Opt. Soc. Am. 72, 156-1603 (1982).

[3] K. H. Womack, "Interferometric Phase Measurement using Synchronous Detection," Proc. SPIE 429 (1983).

[4] W. W. Macy, "Two-dimensional fringe-pattern analysis," Appl. Optics 22, 3898-3901 (1983).

[5] D. W. Robinson, and G. T. Reid, Interferogram Analysis (IOP Publishers, Bristol Ct Philadelphia, 1993).

[6] D. Malacara, M. Servin, and Z. Malacara, Interferogram Analysis for Optical Testing (CRC Press Marcel Decker, New York, 1998).

[7] M. Küchel, "The new Zeiss interferometer," Proc. SPIE 1332, 655-663 (1990).

[8] B. Dörband, W. Wiedmann, U. Wegmann, W. Kübler, and K. R. Freischlad, "Software concept for the new Zeiss interferometer," Proc. SPIE 1332, 664-672 (1990).

[9] D. M. Sykora, and P. de Groot, "Instantaneous measurement Fizeau interferometer with high spatial resolution," Proc. SPIE 8126,812610 (2011).

[10] G. Paez, and M. Strojnik, "Fringe analysis and phase reconstruction from modulated intensity patterns," Opt. Lett. 22, 1669-1671 (1997).

[11] Program: "Phase shifting software PSI Version 6.11," University Erlangen-Nuremberg (2012).

[12] Program: "RAYTRACE Version 0.9," University Erlangen-Nuremberg (2008). 